\title{
Estudios bioarqueológicos del sitio Paso Mayor, sudoeste de la provincia de Buenos Aires
}

RMA

Arqueología

\begin{abstract}
Resumen
En este trabajo se resumen los estudios bioarqueológicos realizados en la colección osteológica del sitio Paso Mayor Y1S2 (partido de Coronel Pringles, Buenos Aires). Los análisis efectuados comprendieron diferentes etapas. En primer lugar se hizo un inventario de la colección y se relevaron algunas variables tafonómicas para conocer el estado general de conservación de los restos. Una segunda etapa estuvo orientada a determinar la estructura sexo-etaria de la muestra. Finalmente se efectuaron estudios de marcadores de estrés ocupacional y el relevamiento de algunas patologías de carácter infeccioso y traumático.

En conjunto estos análisis muestran que en el sitio se inhumaron individuos subadultos y adultos de ambos sexos, los restos fueron enterrados de manera primaria y secundaria. La colección muestra un buen estado de conservación por lo que fue posible determinar la mayoría de los elementos óseos analizados. En cuanto a los estudios de marcadores de actividad física, estos indican la importancia de la actividad deambulatoria y el posible uso de sistemas de armas como el arco y flecha. Se registró una reacción perióstica en los miembros inferiores y un traumatismo en el esqueleto craneal. Finalmente se discute la información bioarqueológica del sitio con la publicada para otros sitios del área.
\end{abstract}

Palabras claves: bioarqueología; cazadores recolectores; región pampeana; área sudoeste; Holoceno tardío.

Bioarchaeological studies of Paso Mayor site, southwest Buenos Aires province

\begin{abstract}
This paper summarizes the bioarchaeological studies of the osteological collection recovered in Paso Mayor Y1S2 site (Coronel Pringles, Buenos Aires). Analyses were carried out in different stages. First, a detailed inventory of the collection was made and some taphonomic variables were considered in order to evaluate the general condition of the bone remains. A second phase was oriented to the determination of the age-sex structure of the sample. Finally, occupational stress markers studies were undertaken along with the observation of certain infectious and traumatic pathologies.

As a whole, these analyses show the primary and secondary burial of sub-adults and adults individuals of both sexes. The collection exhibits a good preservation allowing the determination of most of the analyzed bones. Occupational stress markers show the importance of ambulatory activity as well as the possible use of bows and arrows. Also, a single case of periosteal reaction in the lower limbs and a cranial traumatism were recorded. Finally, bioarchaeological information of the site is discussed and related to the published information of other sites in the area.
\end{abstract}

Key words: bioarchaeology; hunter-gatherers; Pampean region; southwest area; late Holocene.

\section{Introducción. El Registro Bioarqueológico del Sudoeste Pampeano}

El conocimiento sobre las poblaciones pampeanas del Holoceno no es uniforme. Esto se debe a que mientras para algunos períodos el registro bioarqueológico es abundante, otros se caracterizan por la ausencia de restos humanos. También debe tenerse en cuenta que el desarrollo de las investigaciones no ha sido el mismo en las diferentes áreas y que los distintos procesos de formación del registro no han favorecido la conservación de restos óseos en algunas de ellas.

En el caso del sudoeste bonaerense ${ }^{1}$ hasta el momento se conocen 28 sitios con restos humanos (ver Tabla 1), de los cuales sólo 8 poseen fechados radiocarbónicos. El sitio Monte Hermoso 1 es el único que corresponde

\footnotetext{
1 El sudoeste bonaerense es una categoría geográfica que surge de dividir en cuatro cuadrantes a la provincia de Buenos Aires (Campos de Ferreras et al. 2004 en Frontini 2012). Comprende los actuales partidos de Bahía Blanca, Coronel Rosales, Monte Hermoso, Coronel Dorrego, Tornquist, Saavedra, Coronel Suárez, Coronel Pringles, Puán, Patagones y Villarino (Bróndolo y Zinger 1999 en Frontini 2012).
} 
al Holoceno temprano-medio (entre ca. 7800 y 6600 años AP), mientras que el resto abarca el Holoceno tardío (entre ca. 3000 y 500 años AP). Se reconocieron las modalidades de inhumación en 19 sitios; de estos 13 presentan exclusivamente entierros primarios, 3 sólo entierros secundarios y en los otros 3 casos se ven conjugadas las modalidades secundarias junto con las primarias. Para todos los sitios se conoce el número mínimo de individuos enterrados, variando desde sitios donde se halló un único esqueleto hasta sitios como Paso Alsina 1 donde se recuperaron más de 55 individuos, siendo predominantes (61\%) los sitios donde se enterraron menos de 5 individuos (Tabla 1). En 10 sitios los estudios bioarqueológicos permitieron establecer el grupo etario de los individuos enterrados. En 4 de éstos había inhumados tanto adultos como subadultos. En los otros 6 sitios sólo se enterraron individuos adultos. Por el contrario, no se conocen casos donde se hayan inhumado subadultos de manera exclusiva. Finalmente, en 10 sitios se determinó el sexo de los individuos; en 3 sólo se hallaron esqueletos femeninos, en otros 3 esqueletos masculinos y en 4 se recuperaron restos de individuos femeninos y masculinos.

En la Tabla 2 se detallan los diferentes estudios bioarqueológicos llevados a cabo en los sitios del sudoeste pampeano en los últimos años. Estos involucran estudios radiométricos e isotópicos, análisis paleopatológicos tanto del esqueleto craneal como del poscraneal, evaluación de los procesos posdepositacionales, análisis morfométricos en los cráneos y estudios de las características y variabilidad de las prácticas mortuorias.

De la lectura de la Tabla 2 se desprende que los análisis de morfometría craneana fueron los más generalizados y se realizaron en el $28 \%$ de los sitios $(n=9)$. Los estudios paleopatológicos se efectuaron en el $21 \%$ de los casos, estos comprendieron tanto análisis de salud bucal como poscraneanos de señales patológicas de diferente etiología. En el $18 \%$ de los sitios se analizaron las prácticas

\begin{tabular}{|c|c|c|c|c|c|c|}
\hline SITIO & $\begin{array}{l}\text { FECHADO } \\
\text { (años ARCP) }\end{array}$ & $\begin{array}{l}\text { TIPO DE } \\
\text { ENTIERRO }\end{array}$ & $\mathrm{NMI}$ & SEXO & $\begin{array}{l}\text { GRUPO } \\
\text { ETARIO }\end{array}$ & REFERENCIAS \\
\hline Monte Hermoso 1 & $\begin{array}{c}7866 \pm 75 a \\
6606 \pm 79\end{array}$ & - & 2 & - & A & Politis et al. 2009 \\
\hline Cabaña Pirihueico & - & $\mathrm{S}$ & 5 & - & - & Barrientos 1997,2001 \\
\hline Lag. Los Chilenos 1 & $470 \pm 40$ & Py $S$ & 21 & $\mathrm{~F}, \mathrm{M}$ & $A, S$ & Barrientos 1997, Barrientos et al. 2002 \\
\hline Lag. Los Chilenos 2 & - & - & 1 & - & - & Barrientos 1997,2001 \\
\hline Napostá & - & $\mathrm{S}$ & 5 & $\mathrm{~F}, \mathrm{M}$ & $A, S$ & Barrientos 1997,2001 \\
\hline Paso Vanoli & - & - & 6 & - & - & Barrientos 1997 \\
\hline Sauce Chico & - & - & 1 & - & - & Barrientos 1997, 2001 \\
\hline Rinconada & - & - & 9 & - & - & Barrientos 1997, 2001 \\
\hline Villa Sapito & - & - & 1 & - & - & Barrientos 1997; Bayón y Guichón com. pers. \\
\hline Gascón 1 & - & $\mathrm{P}$ & 4 & - & - & Barrientos 1997, 2001 \\
\hline Ybarra & - & - & 5 & - & - & Barrientos 1997, 2001 \\
\hline San Antonio & - & - & 1 & - & - & Barrientos 1997, 2001 \\
\hline La Toma & $2075 \pm 70$ & $\mathrm{P}$ & 1 & - & - & Madrid y Politis 1991 \\
\hline La Petrona & $\begin{array}{c}770 \pm 49 \text { a } 248 \\
288 \pm \frac{ \pm}{2} \pm 49 a\end{array}$ & Py $S$ & 6 & $\mathrm{~F}$ & $A, S$ & Martínez 2010 \\
\hline La Primavera & $\begin{array}{c}2728 \pm 48 \\
570 \pm 40 \text { a } 446\end{array}$ & $\mathrm{P}$ & 5 & M & A & Bayón et al. 2004; Martínez 2010 \\
\hline Paso Alsina 1 & $\begin{array}{c}5 / 0 \pm 40 \text { a } 446 \\
\pm 42\end{array}$ & $S$ & 55 & $\mathrm{~F}, \mathrm{M}$ & $A, S$ & Martínez et al. 2007; Bayala 2008, 2010 \\
\hline Paso Mayor YIS2 & $700 \pm 40$ & Py $S$ & 7 & $\mathrm{~F}, \mathrm{M}$ & $A, S$ & Bayón et al. 2010; Scabuzzo 2010 \\
\hline Ao. Ventana & - & $\mathrm{P}$ & 1 & - & - & Barrientos 1997, 2001 \\
\hline Curamalal & - & $\mathrm{P}$ & 1 & - & - & Barrientos 1997, 2001 \\
\hline Gruta del Esqueleto & - & $\mathrm{P}$ & 1 & - & - & Barrientos 1997, 2001 \\
\hline Lag. del Puán 1 & $3300 \pm 100$ & $\mathrm{P}$ & 1 & M & A & Barrientos 1997; Salceda y Méndez 1990 \\
\hline Lag. del Puán 6 & - & $\mathrm{P}$ & 1 & $\mathrm{~F}$ & A & Barrientos 1997, 2001 \\
\hline Los Huecos & - & $\mathrm{P}$ & 1 & $\mathrm{~F}$ & - & Barrientos 1997, 2001 \\
\hline Saavedra & - & $\mathrm{P}$ & 4 & - & - & Barrientos 1997, 2001 \\
\hline Ao. Las Víboras & - & $\mathrm{P}$ & 2 & - & - & Barrientos 1997, 2001 \\
\hline Abra Agua Blanca & - & - & 1 & - & - & Barrientos 1997, 2001 \\
\hline Laguna del Sauce & - & $\mathrm{P}$ & 10 & - & - & Austral 1968; Bayón com. pers. \\
\hline La Segovia & - & $\mathrm{P}$ & 1 & $\mathrm{M}$ & A & Barrientos 1997, 2001 \\
\hline
\end{tabular}

Referencias: P: primario, S: secundario, F: femenino, M: masculino, A: adulto, S: subadulto Tabla 1. Sitios del sudoeste pampeano con restos óseos humanos.

Table 1. Southwestern pampean sites with presence of human bones. 
mortuorias; estos trabajos incluyen desde descripciones preliminares de las modalidades de entierro hasta trabajos que discuten en profundidad las distintas prácticas de inhumación, su significado y comparación con diferentes sitios del área. La evaluación de los procesos tafonómicos e integridad del registro óseo humano fue realizada en el 14\% de los sitios; en estos casos la cantidad de variables relevadas y la profundidad de los análisis fue heterogénea. El estudio paleodietario a través de isótopos estables se realizó en el 14\% de los casos, los análisis incluyeron valores de $\delta^{13} \mathrm{C}$ tanto de la fracción orgánica como de la inorgánica y de $\delta^{15} \mathrm{~N}$. Finalmente es interesante mencionar que en la mayoría de los sitios (57\%) no se han llevado a cabo ninguno de dichos estudios.

El objetivo de este trabajo es presentar los diferentes análisis bioarqueológicos que se están llevando a cabo en el sitio 2 de la localidad de Paso Mayor (partido de Coronel Pringles, provincia de Buenos Aires, Figura 1). En primer lugar se presentará una síntesis de las características principales de los entierros: descripción de las modalidades de inhumación, sexo y edad de los restos y el relevamiento de variables tafonómicas. Además se detallarán los resultados tanto del análisis de distintos marcadores de actividad física como del relevamiento de algunas patologías de etiología infecciosa y traumática. Finalmente se discutirán aspectos relacionados con las prácticas mortuorias, patrones de actividad y estado de salud de los grupos contextualizándolos con la información bioarqueológica disponible para otros sitios del sudoeste de la región.

\section{El Sitio 2 del Yacimiento Arqueológico I de Paso Mayor}

La localidad de Paso Mayor se ubica en un médano en la margen izquierda del río Sauce Grande (Figura 1). En la década de 1960 un fuerte proceso de erosión eólica dejó al descubierto abundantes y variados restos arqueológicos. Desde el Museo Municipal de Bahía Blanca, el Dr. A. Austral inició trabajos sistemáticos en el lugar y en diversas campañas realizó recolecciones de superficie, excavaciones y delimitó 4 yacimientos. Denominó yacimiento I al ubicado en el médano y dentro de este discriminó dos sitios que corresponden a hoyadas contiguas (Austral 1968). El sitio 1 está en estratigrafía y presenta ocupaciones superpuestas que comienzan en el Holoceno medio. En el sitio 2 efectuó una recolección superficial en la que recuperó diversos vestigios arqueológicos. También ubicó y excavó un conjunto de entierros que nunca fueron publicados y sólo fueron mencionados en el periódico local de Bahía Blanca "La Nueva Provincia" donde se hace referencia "a la presencia de un cementerio indígena...donde

\begin{tabular}{|c|c|c|c|c|c|c|c|}
\hline Sitio & 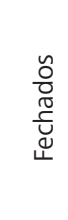 & 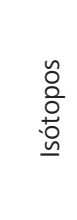 & $\begin{array}{l}\tilde{0} \\
. \frac{U}{\sigma} \\
\frac{0}{0} \\
\frac{0}{0} \\
\frac{0}{0} \\
\frac{d}{\sigma}\end{array}$ & 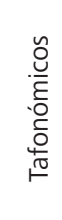 & 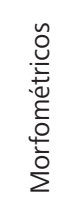 & 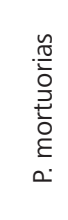 & Referencias \\
\hline Monte Hermoso 1 & X & $x$ & - & - & - & - & Bayón et al. 2011; Politis et al. 2009 \\
\hline Lag. Los Chilenos 1 & $x$ & - & $x$ & $x$ & $x$ & $x$ & $\begin{array}{l}\text { Barrientos 1997; Barrientos et al. 2002; L'Heureaux 2000; } \\
\text { Perez } 2002\end{array}$ \\
\hline Napostá & - & - & $x$ & - & $x$ & - & Perez 2006, L’Heureux 2000 \\
\hline Paso Vanoli & - & - & - & - & $x$ & - & Méndez y Salceda 1989 \\
\hline Gascón 1 & - & - & - & - & $x$ & $x$ & Perez 2002 \\
\hline La Toma & $x$ & - & - & - & $x$ & - & Méndez y Salceda 1989; Politis y Madrid 1991 \\
\hline La Petrona & $x$ & $x$ & $x$ & $x$ & - & $x$ & Torres 2000; Martínez et al. 2009 \\
\hline La Primavera & $\mathrm{X}$ & $X$ & - & - & - & - & $\begin{array}{l}\text { Bayón et al. 2004; Martínez et al. 2009; } \\
\text { Martínez et al. 2007; Martínez et al. 2009; Flensborg }\end{array}$ \\
\hline Paso Alsina 1 & $X$ & $\mathrm{X}$ & $x$ & $x$ & - & $x$ & \\
\hline Paso Mayor YIS2 & $x$ & - & $x$ & $x$ & - & $x$ & Bayón et al. 2010; Scabuzzo 2010 \\
\hline La Segovia & - & - & - & - & $x$ & - & Perez 2002 \\
\hline Lag. del Puán 1 & $x$ & - & - & - & $x$ & - & Perez 2002 \\
\hline Los Huecos & - & - & $x$ & - & - & - & L’Heureux 2000 \\
\hline Saavedra & - & - & - & - & $x$ & - & Méndez y Salceda 1989; Perez 2002 \\
\hline Ao. Las Víboras & - & - & - & - & X & - & Méndez y Salceda 1989; Perez 2002 \\
\hline
\end{tabular}

Tabla 2. Estudios bioarqueológicos efectuados en sitos del sudoeste pampeano.

Table 2. Bioarchaeological studies conducted in pampean Southwestern sites. 


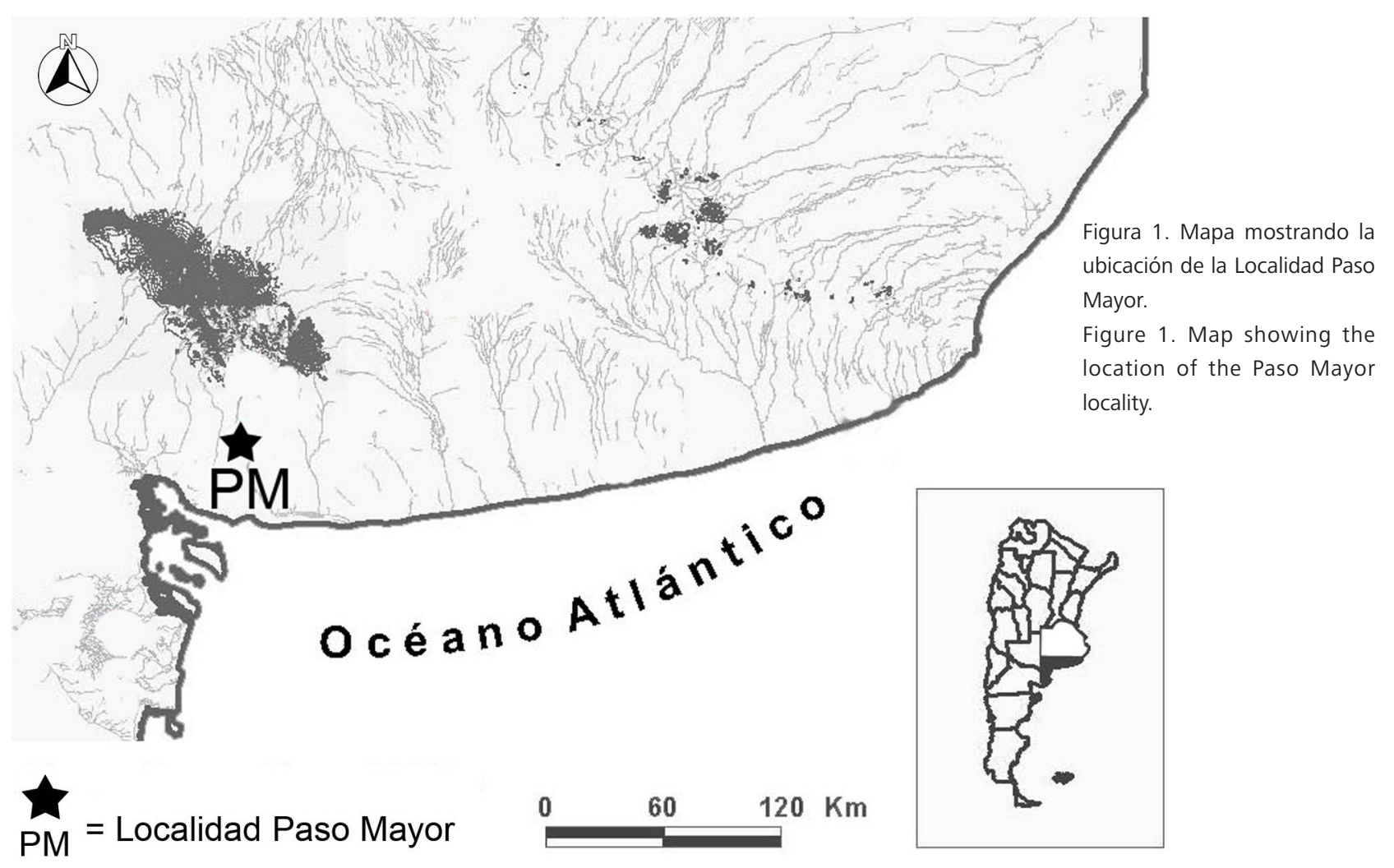

se descubrieron huesos humanos de antigua data junto con flechas, puntas de flecha talladas en piedra" (La Nueva Provincia 1963). Las inhumaciones fueron registradas fotográficamente con mucho detalle, los negativos y copias papel fueron archivados en el Museo y Archivo Histórico Municipal de Bahía Blanca. En la misma institución se conservaron los cuantiosos restos arqueológicos de la localidad.

En superficie, cerca de donde se recuperaron los esqueletos, se encontraron 1054 objetos líticos, 21 tiestos cerámicos, 52 materiales modernos y 44 restos faunísticos, en su mayor parte valvas y cáscara de huevo. A esto debe sumarse un bezoar, una cuenta de valva y fragmentos de colorante. Sin embargo, en la excavación, asociados con los restos humanos no se encontraron artefactos (Vecchi 2011).

En años recientes se retomaron los estudios en Paso Mayor, por una parte se trabajó sobre la colección y por otra se reiniciaron las tareas de campo. La Lic. A. Pupio llevó a cabo el ordenamiento y acondicionamiento de los materiales sobre los que se efectuaron distintos tipos de estudios tecnológicos, zooarqueológicos y bioarqueológicos (Bayón et al. 2006, 2010; Frontini 2012; Scabuzzo 2010; Vecchi 2011). Se hicieron 4 fechados radiocarbónicos sobre distintas muestras, 3 corresponden a restos faunísticos del sitio 1 y el restante se hizo sobre un fragmento de costilla humana (PMY1S2) procedente del sitio 2 (Bayón et al. 2010; Frontini 2012; Scabuzzo 2010). Por otro lado se realizaron tareas de campo que permitieron la reubicación de los sitios 1 y
2. La interpretación efectuada a partir de estos trabajos señala que, tal como indica la secuencia de fechados, el lugar fue recurrentemente ocupado por los cazadores recolectores desde al menos 5900 años $C^{14}$ ARCP hasta el Holoceno tardío final. Esto implica que el paraje ocupaba un lugar importante en la memoria social de los antiguos pobladores (Bayón et al. 2010).

La interpretación conjunta de los datos disponibles indica que, entre 5900 y 3000 años ARCP, la presencia de campamentos residenciales donde se desarrollaron actividades domésticas variadas como la preparación y consumo de animales, en especial ungulados como el guanaco y el venado (Bayón et al. 2010; Frontini 2012). También se manufacturaron artefactos de piedra aprovechando de manera predominante la disponibilidad inmediata de rodados fluviales. En las ocupaciones tardías el lugar también se empleó como base residencial y se llevaron a cabo múltiples tareas, entre ellas el procesamiento y consumo de alimentos de origen animal y posiblemente la manipulación de vegetales, reflejado por la presencia de molinos y manos. En conjunto en ambos sitios se han registrado al menos tres sistemas de armas. Por una parte bolas de boleadora enteras en la colección de superficie y dos preformas en estratigrafía (Vecchi 2011). Las armas con puntas de piedra, de las que se han recuperado más de 90 objetos en superficie, se relacionan con dos sistemas distintos, uno de ellos corresponde a puntas medianas, triangulares y apedunculadas que en estratigrafía se hallaron en los niveles inferiores, estos proyectiles debieron ser arrojados con lanza. Por el contrario, en los niveles superiores se 
encontraron puntas triangulares pedunculadas de tamaño pequeño y muy pequeño que podrían corresponder a sistemas de armas como el arco y flecha (Vecchi 2011). Es durante estas ocupaciones que se realizaron inhumaciones en un área muy cercana al campamento, las cuales se analizan en este trabajo.

\section{Los Estudios Bioarqueológicos: Materiales, Métodos y Resultados}

Como parte de los nuevos trabajos de análisis de la colección se comenzó con los estudios bioarqueológicos de los restos humanos provenientes del sitio 2 de la localidad (Bayón et al. 2010; Scabuzzo 2010). Estos análisis incluyeron varias etapas. En primer término se realizó el estudio de las fotografías que permitieron inferir las modalidades y formas de depositación de los restos humanos. Se estableció la presencia en el sitio de al menos tres inhumaciones diferentes. Una de estas estaba compuesta por un mínimo de tres individuos que conformaban un entierro secundario múltiple, otra consistía en un entierro primario con al menos dos individuos y la tercera inhumación era del tipo secundaria múltiple con dos individuos como mínimo. En cuanto al entierro primario a partir de las fotografías se observó que uno de los individuos se encontraba enterrado en posición lateral derecha con los miembros inferiores flexionados. Se obtuvo un fechado radiocarbónico de los entierros de $700 \pm 40$ años ARCP (AA-56780, Bayón et al. 2010).

La segunda etapa involucró la realización del inventario básico de las partes presentes, el cálculo del número mínimo de individuos y la determinación de sexo y edad de los restos. También se relevaron algunas variables tafonómicas (presencia de marcas de raíces, depositaciones químicas tales como óxido de manganeso y carbonato de calcio, presencia de fisuras, marcas de roedor y completitud de los elementos). Finalmente se efectuó el análisis de los marcadores de estrés ocupacional. Específicamente se relevaron las señales de osteoartritis y el desarrollo de las zonas de inserción muscular. Esto tuvo como fin evaluar cuáles de las áreas articulares y de las zonas de inserción muscular estuvieron expuestas a mayor estrés. Además esta etapa involucró el estudio de algunas patologías de etiología infecciosa y traumática. En este punto es importante aclarar que los estudios se realizaron considerando al elemento como unidad de análisis y no a los individuos. Esto se debe, por un lado, a que la mayoría de los elementos provenían de entierros secundarios y por el otro, a que los restos fueron guardados sin conservar la unidad por entierro o individuo.

Determinación sexo etaria de la muestra y estimación del NMI

En la primera etapa de trabajo se analizaron un total de 128 restos óseos, la mayoría de estos pertenecientes al esqueleto poscraneal, sobre todo huesos largos de los miembros superiores e inferiores (Bayón et al. 2010), siendo muy baja la frecuencia de coxales, sacro, rótulas, tarsos, escápulas, clavículas, falanges, vértebras y costillas (Figura 2).

A partir del análisis de distintos huesos largos se pudo estimar para el conjunto un número mínimo de siete individuos. De estos, seis fueron determinados como adultos (mayores de 20 años) sin que se pudiera precisar si eran adultos jóvenes, medios o mayores y un individuo como subadulto (entre 7 y 10 años de edad). La diferenciación de los restos en adultos y subadultos se efectuó a partir de criterios cualitativos (estado de fusión de las epífisis y de los elementos que componen los coxales) y de la longitud de la diáfisis de los huesos largos (Bass 1995; Buikstar y Ubelaker 1994).

El sexo probable de los individuos adultos se determinó a partir de los coxales y de los cráneos siguiendo los criterios bioarqueológicos estándar (Buikstra y Ubelaker 1994). En el caso de los coxales se observó la profundidad y el ancho de la escotadura ciática mayor, las características de la rama isquiopúbica, la morfología del ilion y la concavidad subpúbica. En el cráneo se observó el grado de desarrollo de la cresta nucal, de las arcadas supraorbitarias y de los procesos mastoideos (Bass 1995; Buikstra y Ubelaker 1994; White y Folken 2005). La presencia de partes diagnósticas en cinco coxales permitió reconocer que

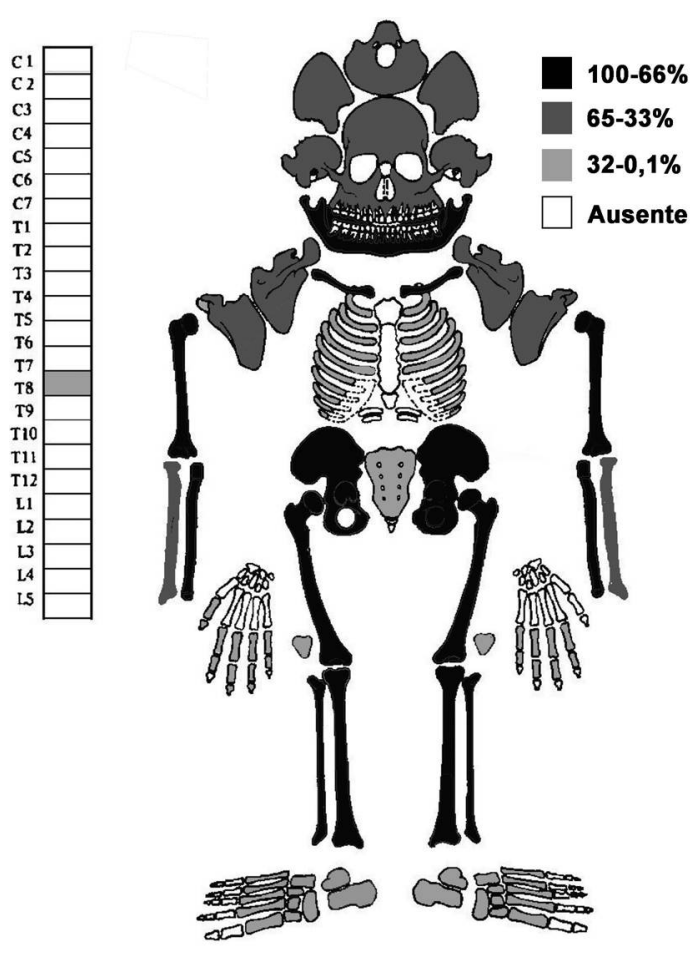

Figura 2. MAU\% de los restos óseos humanos provenientes del sitio 2 de la localidad Paso Mayor.

Figure 2. \%MAU of human skeletal remains from the site 2 of the Paso Mayor locality. 
el conjunto se componía de individuos femeninos y masculinos. Tres de los coxales han sido asignados a individuos femeninos y dos a individuos masculinos. De los cuatro cráneos, dos presentaron características masculinas y los otros dos femeninas. En síntesis, a partir de estos estudios bioarqueológicos se pudo establecer que en el sitio fueron inhumados tanto individuos adultos como subadultos, entre los adultos hay individuos masculinos, femeninos e indeterminados (Bayón et al. 2010).

Con el fin de conocer el estado general de conservación del conjunto, se llevó a cabo el relevamiento de algunas variables tafonómicas: presencia de marcas de raíces, depositaciones químicas tales como óxido de manganeso y carbonato de calcio, presencia de fisuras, marcas de roedor y completitud de los elementos, tomando en cuenta una escala de 4 grados (Guichón et al. 2000). La colección en general presenta un buen estado de conservación. La variable que más afectó al conjunto fue la presencia de raíces (83\% de los elementos), seguida por las manchas de óxido de manganeso (59\% de los huesos), la presencia de carbonato de calcio en la superficie no se detectó en ningún caso. Por último, se registraron marcas de roedor en un único elemento. En cuanto a la completitud de los huesos, de los restos analizados 91 $(79,8 \%)$ han sido ubicados en las categorías 3 o 4 de completitud, es decir representados por más del 50\% del total del elemento. El alto grado de completitud de los huesos explica en gran parte que haya sido posible identificar la mayoría de ellos. Se pudo determinar que de los 128 restos analizados el 68,7\% presentaba fisuras en la superficie cortical (Bayón et al. 2010). Se observó pigmento en el $30 \%$ de los elementos óseos analizados, esto fue relevado en distintos huesos correspondientes a individuos adultos tanto de sexo femenino como masculino. Por el contrario, no se observó ningún elemento de subadulto con ocre. Esta diferencia entre adultos y subadultos podría deberse a un tratamiento diferencial de los restos de los individuos de las distintas categorías etarias, aunque no puede descartarse que se deba a que con el paso del tiempo la pintura haya desaparecido. Finalmente, en uno de los cráneos correspondientes a un adulto masculino se detectó la presencia de deformación craneana del tipo tabular erecta (Bayón et al. 2010; Scabuzzo 2010).

\section{Marcadores de estrés ocupacional}

En la década de 1990, los estudios de marcadores de estrés ocupacional se afianzaron a nivel mundial como una herramienta útil para acceder a las actividades físicas cotidianas y para resolver otros problemas de interés arqueológico. Algunos de los temas que fueron abordados a partir de estos análisis fueron la diferencia de las exigencias físicas entre los miembros de un grupo (Neves 1984; Rodrigues Carvalho 2004), la división sexual de las tareas (Merbs 1983; Mendonça de Souza 1992), los cambios en las demandas mecánicas al pasar de un modo de subsistencia a otro (Bridges 1992), las variaciones en el uso del cuerpo en el proceso de hominización (Jurmain 1980), el uso de diferentes tecnologías y en algunos casos para la reconstrucción actividades especificas (Kennedy 1989; Capasso et al. 1999 entre otros).

Como ya se mencionó, una tercera etapa de los estudios bioarqueológicos realizados en la colección comprendió el relevamiento de dos tipos de marcadores de estrés físico: osteoartritis y desarrollo de las zonas de inserción muscular. Estos estudios tuvieron como fin conocer cuáles de las zonas articulares y áreas de inserción muscular estuvieron sometidas a mayor estrés mecánico. Los análisis se hicieron en aquellos elementos poscraneales pertenecientes a individuos adultos y con una buena preservación (ver Scabuzzo 2010, 2012). Como en general el estado de conservación de los huesos largos es bueno, se pudieron llevar a cabo estos estudios en un porcentaje alto de los elementos óseos. Es interesante mencionar que en el relevamiento de los marcadores físicos se tomó como unidad de análisis los elementos óseos y no los individuos (Scabuzzo 2010).

En el caso de la osteoartritis, se analizaron todas las áreas de articulación de los huesos. El procedimiento para las observaciones fue: en los huesos largos, metacarpos y metatarsos se registró por separado cada epífisis. En los huesos del carpo, del tarso y las falanges en cambio, no se discriminó entre las carillas articulares. En las vértebras, se separó el cuerpo vertebral del arco (siguiendo a Mendonça de Souza 1992). En el sacro se diferenciaron las alas laterales del promontorio y las carillas de articulación con la quinta vértebra lumbar. Para los huesos planos (cadera y escápula) se relevó el acetábulo y la cavidad glenoidea (Scabuzzo 2012). Siguiendo las recomendaciones de Neves (1984) y Rodrigues Carvalho (2004), las afecciones fueron clasificadas en cuatro grados de severidad (ausente, leve, moderado y severo).

De los 114 restos poscraneales que componen la colección, 73 (64\%) fueron aptos para relevar las señales de osteoartritis, totalizándose un relevamiento de 98 áreas articulares. La mayoría corresponden a huesos largos, siendo los más representados los húmeros, los fémures y los cúbitos.

De los 73 elementos analizados el diagnóstico macroscópico reveló señales de la patología en tres elementos $(4,1 \%)$ : un coxal derecho probablemente femenino y dos escápulas. En el primer caso se presentan labiaciones en la cavidad acetabular y porosidades en forma de coalescencia restringidas (Buikstra y Ubelaker 1994). Por otro lado, en una escápula izquierda y otra derecha correspondientes a distintos individuos se determinó la presencia de lesiones de forma redondeada en la cavidad glenoidea, modificaciones atribuibles a una osteocondritis articular del hombro (Figura 3). Este tipo de modificación articular fue relacionada con movimientos 
de extensión del brazo contra una fuerza compresiva en dirección al hombro (Capasso et al. 1999). Autores como Merbs (1983) atribuyen estas modificaciones a actividades tales como el uso de arco y flecha cuando la modificación se presenta de manera unilateral y con actividades como remar en kayak cuando la lesión se presenta de manera bilateral. Las características del material y el análisis realizado a nivel de elemento y no de individuo limitan las afirmaciones respecto de si el estrés fue bilateral o unilateral, no obstante una de las escápulas derecha que por el tamaño y las características parece corresponder al mismo individuo no presentó este tipo de patología. Lo que parece indicar que la lesión fue unilateral. La baja frecuencia de señales de osteoartritis en la colección es un tema que será discutido más adelante.

\section{FIGURA 3.}

El relevamiento de los marcadores músculo-esqueletales consistió en la inspección macroscópica de 15 zonas de inserción muscular elegidas previamente, 8 en los miembros superiores (pectoral mayor, deltoides, braquirradial, supinador, bíceps, tríceps, braquial, pronato cuadrado) y 7 en los inferiores (glúteo mayor, glúteo menor, glúteo medio, aductores de la pierna, cuádriceps, sóleo y plantares). La selección de las inserciones a relevar tuvo en cuenta las áreas de fácil ubicación en el hueso, las que participan de los principales movimientos del cuerpo y las que aparecen con mayor frecuencia nombradas en la literatura (Scabuzzo 2010, 2012). Las áreas de inserción muscular fueron analizadas siguiendo a Hawkey y Merbs (1995) y Hawkey (1998), considerando tres aspectos diferentes: la robusticidad, las lesiones de estrés y las osificaciones. Mientras la robusticidad hace referencia al desarrollo normal de las zonas de inserción muscular, las osificaciones y lesiones de estrés son rasgos asociados con traumas a nivel de las éntesis. Cada uno de estos aspectos fue clasificado en una escala de intensidad creciente desde el grado 0 al grado 3 (Scabuzzo 2010).

Se examinaron 42 elementos poscraneales, de los cuales 17 (40\%) son huesos de los miembros inferiores y 25 (60\%) corresponden a los miembros superiores, totalizándose 102 áreas de inserción muscular relevadas. Del análisis de las zonas de inserción en conjunto, sin discriminar entre miembros superiores e inferiores se determinó que los grados de robusticidad que predominan son los moderados seguidos de los severos. En muy pocos elementos la robusticidad de las inserciones fue nula (Scabuzzo 2010).

Sin embargo, un análisis más detallado de los datos discriminando entre miembros superiores e inferiores muestra una diferencia en los grados de robusticidad predominantes. Mientras que en las inserciones musculares superiores los desarrollos leves (35\%) son seguidos de los moderados (32\%), en las inserciones musculares de las piernas tienden a dominar los grados moderados (48\%) seguidos de los severos (40\%).

Asimismo en los miembros inferiores se detectaron los tres casos de traumas a nivel de las inserciones. Dos de estos corresponden a lesiones de estrés en la parte posterosuperior de la tibia en la inserción del músculo sóleo, las que aparecieron en un elemento derecho y en uno izquierdo que parecen corresponder al mismo individuo. El otro caso es una osificación leve en la parte posterior de un fémur derecho donde inserta el glúteo mayor.

Del análisis de cada zona de inserción muscular,

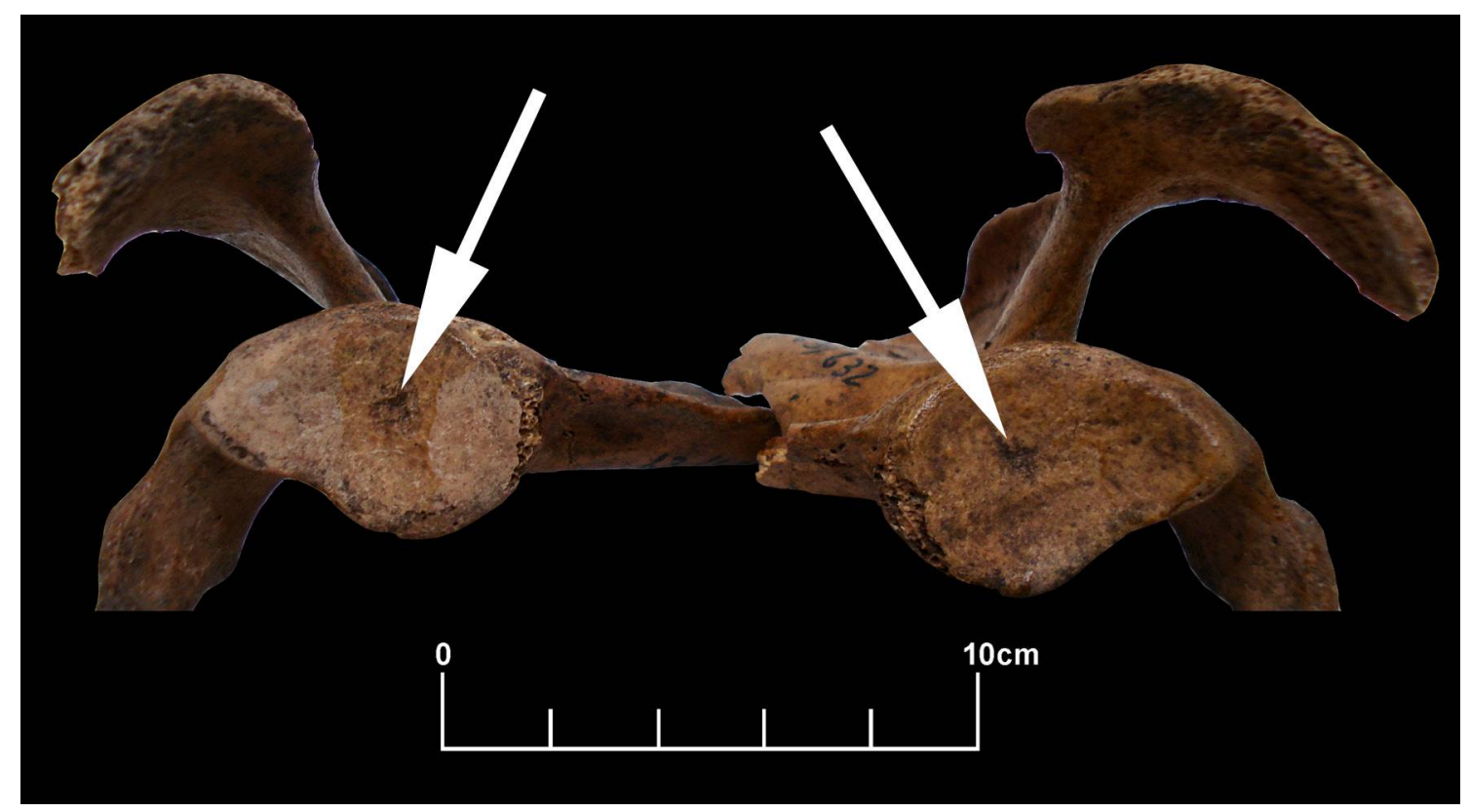

Figura 3. Escápula derecha e izquierda con signos de osteocondritis en la cavidad glenoidea.

Figure 3. Right and left scapula with signs of osteochondritis in the glenoid cavity. 
discriminando entre miembros superiores e inferiores, se desprende que en los miembros superiores los músculos más desarrollados fueron el bíceps seguido por el pectoral mayor y el braquial. El bíceps y el braquial trabajan en conjunto en los movimientos de flexión del codo, acercando el antebrazo al brazo. Por su parte, el pectoral mayor participa de los movimientos que involucran la aducción y flexión del brazo.

Finalmente, es de destacar la presencia de lesiones de estrés en una clavícula izquierda, la cual muestra una oquedad de aproximadamente $1 \mathrm{~cm}$ de diámetro localizada en la zona de inserción del ligamento costoclavicular. Se trata del único caso de lesión de estrés registrado en miembros superiores y el único detectado en el conjunto de las clavículas.

Del análisis de las áreas de inserción del miembro inferior se infiere que los músculos más desarrollados fueron los que se fijan en la línea áspera, los cuadriceps y el glúteo mayor. En conjunto estos músculos participan de movimientos de aducción, rotación y extensión de la pierna, estando todos ellos comprometidos en los movimientos de deambulación.

\section{Otros marcadores de actividad física y patologías}

Como parte de la inspección de los restos se halló un caso de marcador de estrés postural en uno de los elementos.
Si bien el relevamiento de este tipo de modificaciones no se hizo de manera sistemática es interesante el caso observado. Se trata de una oquedad o faceta que se presenta en la parte posterior del fémur, encima del cóndilo lateral (Figura 4a). Este tipo de modificación fue vinculada con la posición de acuclillarse y ha sido ampliamente documentada por varios investigadores en distintos sitios (Capasso et al. 1999; Molleson 1994; Santiago et al. 2011; Ubelaker 1979).

También se constataron evidencias de traumas en uno de los cráneos analizados, perteneciente a un adulto masculino. En primera instancia, se identificó una depresión de forma oval de aproximadamente $1,25 \mathrm{~cm}$ de diámetro, localizada en el parietal izquierdo a 0,5 $\mathrm{cm}$ de la sutura lámbdica. La misma muestra bordes redondeados y la presencia de poros en la superficie. Estas características permitieron relacionarla con un proceso traumático y se descartó su vinculación con algún tipo de agente posdepositacional. La presencia de señales de remodelación ósea indica que el individuo sobrevivió luego del impacto (Florencia Gordón com. pers. 2009, Scabuzzo 2010).

Por otro lado, en el mismo cráneo sobre el parietal izquierdo y a unos centímetros de la lesión anteriormente mencionada se registró una marca de corte. La misma mide aproximadamente $3 \mathrm{~cm}$ de longitud. Este trauma se caracteriza por un fondo recto con depositación de

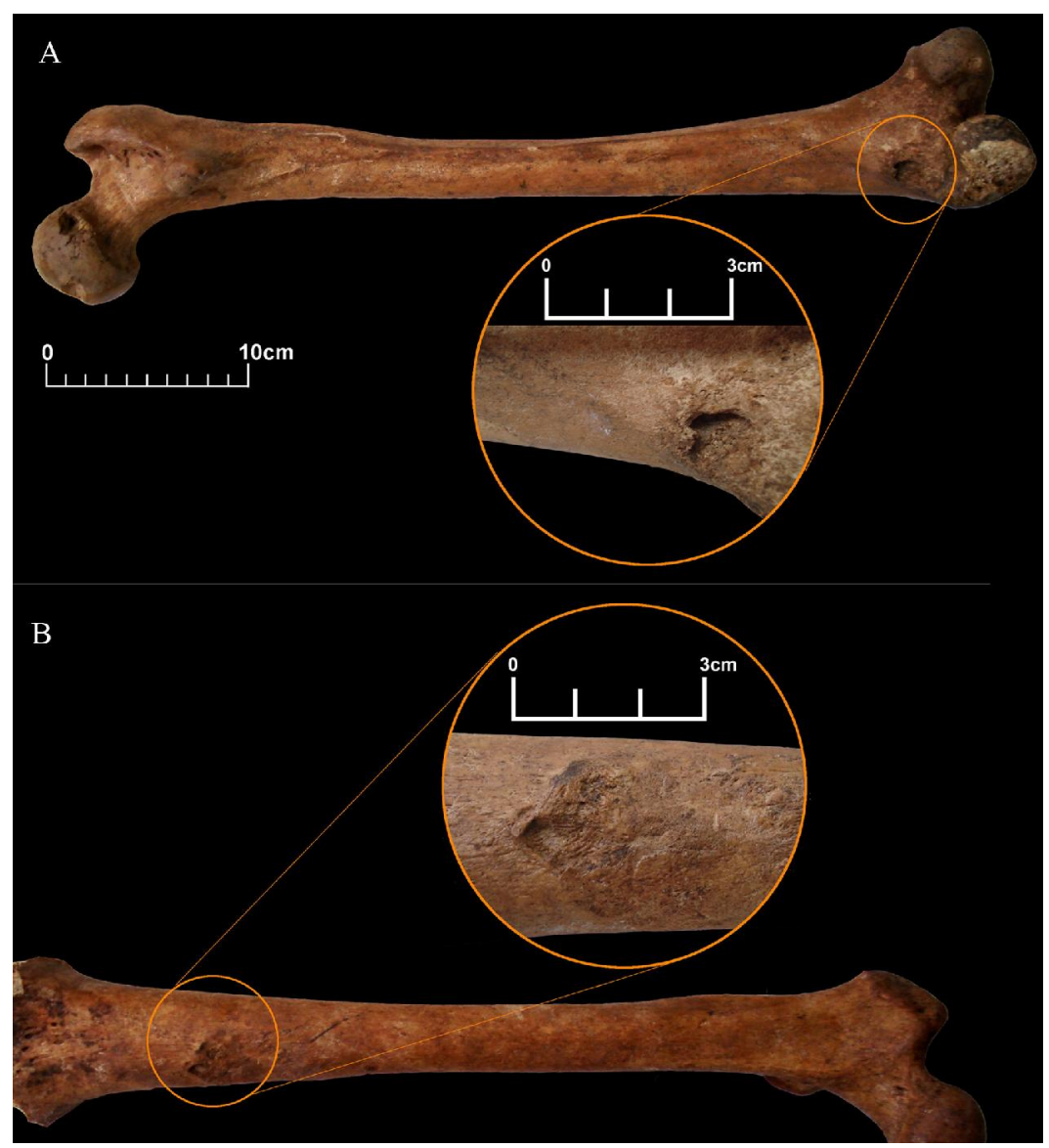

Figura 4a. Faceta accesoria posiblemente relacionada con la postura de acuclillado.

Figure 4a. Accessory facet possibly related to squatting position.

Figura 4b. Reacciones periósticas a nivel de la diáfisis distal del fémur (vista anterior).

Figure $4 b$. Periosteal reactions at the distal femoral shaft (front view). 
óxido de manganeso y pequeñas lascas óseas adheridas (Florencia Gordón com. pers. 2009, Scabuzzo 2010), no se observaron signos de remodelación ósea asociados, lo que indicaría que fue realizada en momentos cercanos a la muerte del individuo.

Finalmente, se identificaron reacciones periósticas, en la diáfisis distal de un fémur, sobre su cara anterior (Figura 4b). Este tipo de reacciones son consideradas como manifestaciones de infecciones o heridas (Ortner y Putschar 1981) y es una de las señales patológicas de mayor aparición en los esqueletos provenientes de sitios arqueológicos. Todavía no se realizaron radiografías sobre el elemento para determinar si la infección afectó al tejido cortical solamente o también se encuentra comprometida la médula ósea.

\section{Discusión}

El estudio de la colección permitió generar información nueva en cuanto a sexo, edad, partes presentes, cronología, procesos tafonómicos y aportar nuevos datos sobre los entierros secundarios tardíos en la región pampeana. Además se obtuvo información novedosa sobre algunos marcadores de estrés ocupacional que permitieron realizar inferencias en relación con los patrones de actividad de los cazadores recolectores de este período (Scabuzzo 2010). Sin embargo el estudio de la colección tuvo limitaciones debido a que gran parte de la información de campo se perdió (e.g organización de los elementos en los entierros secundarios, presencia de objetos asociados) y no fue posible recuperarla una vez que los esqueletos fueron extraídos del terreno, a lo que se suma que no se tuvo acceso a las libretas de campo.

Dos aspectos vinculados con prácticas sociales resultan interesantes en el caso del sitio bajo estudio: una es la presencia de deformación craneana y la otra son las prácticas mortuorias. En trabajos previos se han discutido ambos aspectos (Bayón et al. 2010; Scabuzzo 2010). El tipo de deformación craneana tabular erecta fue asignada por Baffi y Berón (1992) y Barrientos (1997) al Holoceno tardío final y se encuentra ampliamente representada en sitios de este período del sudeste bonaerense (Barrientos 1997), valle inferior del rio Colorado (Martínez y Figuerero Torres 2000; Martínez et al. 2007), valle inferior del rio Negro (Bernal et al. 2008) y La Pampa (Baffi y Berón 1992). En el sitio bajo estudio sólo uno de los individuos presentó el cráneo deformado, lo que llevó a que en trabajos previos se postularan dos posible hipótesis. Por un lado se planteó que el individuo pertenecía a otro grupo de origen, y como una segunda alternativa, que por algún motivo se haya dado un tratamiento diferencial a este individuo dentro del grupo (Bayón et al. 2010; Scabuzzo 2010). Sin embargo, no hay que descartar que haya un sesgo por el tamaño reducido de la muestra.

A lo largo del Holoceno tardío, la presencia de entierros secundarios en sitios de la región pampeana ha sido un rasgo frecuente y que tendió a enfatizarse a partir de los 1000 años AP. Para el área de estudio, una tendencia clara se observa a partir del análisis de los siete sitios que poseen información radiocarbónica y de modalidades de entierro (ver Tabla 1). En aquellos sitos correspondientes al Holoceno tardío inicial (entre 3000-1000 años AP) sólo se registró la modalidad de inhumación primaria, en tanto todos los sitios del Holoceno tardío final presentan entierros secundarios, en algunos casos como único tipo de inhumación (Paso Alsina 1) y en otros acompañados de las modalidades primarias (Paso Mayor Y1S2, Los Chilenos 1, La Petrona). Por lo tanto a nivel areal la presencia de entierros secundarios parecería ser propia del Holoceno tardío final. Esto contrasta con la información disponible para el área interserrana bonaerense donde este tipo de inhumaciones se da desde el Holoceno medio (Scabuzzo y Politis 2011).

Otro aspecto interesante a destacar es la presencia en el sitio de esqueletos tanto de adultos como de subadultos, de sexo femenino como masculino. Esto indica que el lugar fue utilizado para la inhumación de distintos grupos etarios y de ambos sexos y que no hubo una segregación del área de entierro para algún grupo en particular sino que su uso fue generalizado. En contraste es interesante señalar que en el sudoeste bonaerense hay sitios donde se inhumaron distintos grupos erarios y ambos sexos como en Paso Mayor y sitios donde sólo se enterraron adultos de sexo femenino (La Petrona, Martínez 2010).

En relación con los patrones de actividad física el registro arqueológico de la localidad nos brinda el marco con el cual evaluar, en términos de acción, las modificaciones observadas en los esqueletos. En este caso de estudio la puesta en diálogo del registro arqueológico con el registro bioarqueológico permite fortalecer las inferencias realizadas sobre las actividades físicas y modos de vida de los cazadores recolectores pampeanos del Holoceno tardío.

En cuanto a los marcadores de estrés muscular, es importante destacar que este tipo de estudios no se realizó en ningún sitio de la región correspondiente al Holoceno tardío, por lo que no hay datos regionales con los cuales comparar los resultados aquí obtenidos. En primera instancia cabe mencionar el predominio de grados moderados y severos de desarrollo muscular y la baja frecuencia de zonas de inserción con ausencia de robusticidad. Además fueron registrados pocos casos de lesiones patológicas a nivel de las inserciones musculares. Esto indicaría que, en general, hubo un uso intensivo de la musculatura y en pocos casos las altas exigencia musculares sobrepasaron los límites de reacción del hueso, ocasionando patologías en las zonas de inserción en forma de oquedades u osificaciones (Scabuzzo 2010).

Por otro lado, es notable el mayor desarrollo de los 
músculos que mueven los miembros inferiores en comparación con los músculos que accionan los brazos y los hombros. Este dato está mostrando un significativo empleo y demanda de la musculatura ligada con la movilidad de los individuos. Esto se encuentra apoyado por la mayor frecuencia de lesiones en las inserciones que mueven muslos y piernas. En el miembro inferior, los músculos que mayor desarrollo presentaron en toda la serie fueron el glúteo mayor y el cuádriceps. El primero de estos trabaja en la extensión del muslo y fue vinculado con el movimiento de corrida o marcha en terrenos accidentados, también se destaca su participación en el mantenimiento de las posturas erguidas. El cuádriceps junto con la acción de otros paquetes musculares participa de actividades como la caminata debido a que su función principal es la de extensor de la rodilla. Liberman y colaboradores (2006) enfatizaron el papel central de este músculo en la actividad de correr.

En este sentido, la actividad deambulatoria se encuentra fuertemente señalada por los marcadores músculoesqueletales aquí analizados, esto resulta concordante con las evidencias aportadas por las materias primas líticas que indican un amplio radio de circulación para los grupos asentados en el valle medio del río Sauce Grande (Bayón et al. 2006). El acarreo de las rocas se hizo de manera frecuente, por más de $300 \mathrm{~km}$ en el caso de Tandilia y por $40 \mathrm{~km}$ en el caso de la costa atlántica.

En los miembros superiores, el gran desenvolvimiento de músculos como el pectoral mayor y el deltoides, involucrados en los movimientos del hombro y el brazo fue relacionado por distintos autores con actividades tales como el cuereo de animales, el manejo de machetes, la percusión de materias primas líticas, así como cortar madera, cavar y lanzar objetos (Capasso et al. 1999; Chapman 1997; Hawkey y Merbs 1995; Steen y Lane 1998; Teixeira 2004).

Otra de las inserciones que mostró gran demanda mecánica fue el bíceps, cuya función principal es de flexor del brazo y de manera secundaria supinación cuando los codos están flexionados. El desarrollo del mismo fue relacionado con tareas como el uso de remos dobles, carga de peso con los codos flexionados y uso de arco y flecha (Rodrigues Carvalho 2004). Finalmente el braquial actúa de manera sinérgica con el bíceps en los movimientos de flexión del codo. Por otro lado, el acentuado desarrollo de músculos como el pronato cuadrado indica que los movimientos de pronación y supinación de los antebrazos fueron frecuentes. La actuación de estos músculos en una gran cantidad de actividades no hace fácil que relacionemos su hipertrofia con la ejecución de actividades específicas (Rodrigues Carvalho 2004). Sin embargo, es posible conocer a través del registro arqueológico la realización de una serie de acciones físicas que en gran parte podrían explicar la demanda y el uso de estos grupos musculares. A partir del análisis de los materiales de los niveles superiores del sitio 1 fue posible interpretar que los grupos que habitaron este campamento cotidianamente hacían diferentes tareas tales como manufactura de instrumentos líticos, procesamiento y traslado de vegetales y animales (Bayón et al. 2010; Frontini 2012). Una gran cantidad de artefactos fueron hechos y utilizados en el lugar. Los modos de reducción de las rocas fueron la talla por percusión directa, la talla bipolar, el picado y la abrasión. Por otro lado, el registro arqueofaunístico dio cuenta de la caza, trozamiento y traslado de herbívoros de tamaño grande como el venado y el guanaco (Frontini 2012). Algunas de estas tareas fueron ejecutadas con el uso predominante de un brazo en tanto otras necesitaron el empleo simultáneo de ambos brazos. En el caso particular de Paso Mayor por la historia de la colección no se pudo saber si el desarrollo de las inserciones se dio de manera unilateral o bilateral o si hubo diferencias entre los individuos en el desenvolvimiento de estas áreas musculares, por ello no se pudo determinar, en estos casos, el desempeño de una actividad específica sino sólo mencionar el rango posible de tareas que pudieron ser efectuadas.

En cuanto a los análisis de osteoartritis, en primer lugar cabe destacar la baja frecuencia de señales de degeneración articular en los elementos, sólo se observaron signos de la enfermedad en un coxal posiblemente femenino y en dos escápulas. Esta baja prevalencia (4,1\% de los elementos) contrasta con lo esperado para grupos con modos de vida cazador recolector (Larsen 1997). En el caso de los estudios de carácter paleopatológico realizados en otros sitios de la región pampeana con cronología semejante como Paso Alsina 1 (Flensborg 2008, 2010) y Chenque I (Luna 2008), estos ambos sitios mostraron una frecuencia algo mayor de elementos con signos de la enfermedad. Para el primero de estos sitios Flensborg (2010) determinó que la osteoartritis es la patología de mayor prevalencia en la muestra, presente en el $28,7 \%$ de los huesos poscraneales analizados, siendo las vértebras los elementos más afectados. Por su parte, Luna (2008) en el sitio Chenque I también observó que la porción anatómica más comprometida es la columna, con una prevalencia del 11,36\%. En Paso Mayor la incidencia de esta patología es la más baja y la discrepancia con los otros sitios de la región pude ser explicada por distintos argumentos. En primer lugar, el tamaño de la muestra analizada puede explicar la baja frecuencia de osteoartritis. En segundo lugar se puede argumentar que las poblaciones que habitaron el sitio estuvieron expuestas a un menor estrés mecánico que se tradujo en una baja proporción de articulaciones afectadas. Hasta el momento es imposible decidir cual de estas alternativas es la más probable. Futuros análisis en colecciones de cronología semejante ayudarán a resolver este interrogante (Scabuzzo 2010).-

De los pocos elementos con degeneración articular se destacan dos escápulas, en las cuales se observó una 
osteocondritis en la cavidad glenoidea, este tipo de modificación unilateral fue vinculada con el uso del arco y flecha. El contexto arqueológico, con presencia de puntas triangulares pequeñas provenientes de los niveles superiores del sitio 1, permite relacionar de manera tentativa la lesión registrada en el hombro con el uso habitual y rutinario de dicho sistema de armas. Además el gran desarrollo del bíceps y del braquial también puede ser vinculado en parte con la flexión realizada por los brazos al impulsar este tipo de proyectiles. En cuanto al estudio de las patologías, en un fémur se registraron reacciones periósticas. Esta baja frecuencia de infecciones inespecífica no es concordante con lo observado por Barrientos (1997) en sitios pampeanos con cronología semejante (Laguna Los Chilenos 1). Por su parte, en Paso Alsina 1 Flensborg (2010) no encontró casos de patologías infecciosas en los elementos analizados. En el caso de Paso Mayor, las características de la muestra y el pequeño tamaño de la misma pueden ser factores que actúen sesgando estos resultados.

Por otro lado, se observaron dos lesiones traumáticas en uno de los cráneos correspondiente a un individuo adulto de sexo masculino. Una de las lesiones es una fractura en depresión en el parietal izquierdo que muestra señales de remodelación ósea lo que indicaría que no fue la causa de muerte del individuo o al menos que el individuo sobrevivió un tiempo luego de que se produjo el golpe (Florencia Gordón comp. pers. 2009). Este tipo de lesiones aparecen frecuentemente en los restos humanos de sitios arqueológicos y los datos forenses actuales relacionan este tipo de heridas con los golpes producidos por armas contundentes como martillos (Lessa 2004). En la región pampeana sólo se registraron cráneos con lesiones semejantes a la encontrada en Paso Mayor, en el sitio La Toma (pdo. Coronel Pringles) correspondiente al Holoceno tardío que fueron atribuidas al impacto de bolas de boleadoras (Politis 1984; Politis y Madrid 2001). Para el norte de Patagonia, Barrientos y Gordón (2004) mencionan la presencia de este tipo de traumatismos en cráneos procedentes de sitios arqueológicos ubicados en los ríos Negro y Chubut, correspondientes al Holoceno tardío final.

La otra lesión registrada corresponde a un corte localizado a escasos centímetros de la fractura mencionada. La ausencia de señales de reacción ósea vinculadas con esta marca hace suponer que la misma se produjo en momentos perimortem, y que no es contemporánea con el otro traumatismo. Sin embargo, uno de los temas que queda por explorar es si el corte tiene algún tipo de relación con la preparación de los entierros secundarios; de ser así el mismo no sería producto de un trauma ocasionado en el individuo vivo.

En síntesis, el estudio de los restos óseos humanos del sitio Paso Mayor permitió aportar información referida a distintos aspectos de los modos de vida de los cazadores recolectores que habitaron el sudoeste pampeano en el Holoceno tardío. La comparación de los resultados de estos análisis con los obtenidos por otros investigadores mostró una alta variabilidad en la prevalencia de las patologías, sin poder hasta el momento establecer patrones generales. En cuanto a los marcadores de estrés ocupacional este tipo de estudios no ha sido aún muy aplicado en los estudios bioarqueológicos por lo que la posibilidad de comparación es todavía muy limitada. Finalmente, las interpretaciones efectuadas en relación con los patrones de actividad física deben ser tomadas con precaución debido al reducido tamaño que presenta la muestra y a que el análisis fue hecho a nivel de elemento y no de individuo.

La Plata, 10 de agosto de 2012

\section{Agradecimientos}

Este trabajo fue realizado en el marco de mi tesis doctoral y con el apoyo económico del PICT Bicentenario 1517 (dirigido por I. González) y SECyT UNS 24/I182. Quiero agradecer a Alejandra Pupio y Cristina Bayón por darme la posibilidad de trabajar con los materiales osteológicos del sitio. También al Museo Municipal de Bahía Blanca por su gentileza y buena predisposición para llevar a cabo la investigación, a Romina Frontini y Rodrigo Vecchi. A Celeste Weitzel por su ayuda en la preparación del manuscrito. Finalmente a mis directores Ricardo Guichón y Gustavo Politis por las sugerencias y lectura previa de este trabajo. Sin embargo el contenido es absoluta responsabilidad de la autora.

\section{Bibliografía}

Austral, A. 1968. Los hallazgos arqueológicos de Paso Mayor II. Partido de Coronel Pringles, Prov. de Bs. As. Cuadernos del Sur, 8-9:1-32.

Baffi, I., M. Berón. 1992. Los restos óseos humanos de Tapera Moreira (La Pampa), y la deformación artificial en la Región Pampeana. Análisis tentativo. Palimpsesto, 1: 25-36.

Barrientos, G. 1997. “Nutrición y dieta de las poblaciones aborígenes prehispánicas del sudeste de la Región Pampeana". Facultad de Ciencias Naturales y Museo, Universidad Nacional de La Plata, Argentina, 231 pp. Tesis Doctoral inédita. Biblioteca de la Facultad de Ciencias Naturales y Museo.

Barrientos, G. 2001. Una aproximación bioarqueológica al estudio del poblamiento prehispánico tardío del Sudeste de la Región Pampeana. Intersecciones en Antropología, 2:3-18.

Barrientos, G. y F. Gordón. 2004. Explorando la relación entre nucleamiento poblacional y violencia interpersonal durante el Holoceno tardío en el noreste de Patagonia 
(República Argentina). Magallania, 32:53-69.

Barrientos, G., F. Oliva y M. Del Papa. 2002. Historia pre y postdepositacional del entierro secundario del sitio laguna Los Chilenos 1 (provincia de Buenos Aires). Relaciones de la Sociedad Argentina de Antropología, XXVII: 303-325.

Bass, W. 1995. Human Osteology: A Laboratory and Field Manual. 4th ed. Special Publication $N^{\circ} 2$. Missouri Archaeological Society, Columbia.

Bayala, P. 2008. "El registro bioarqueológico del sitio Paso Alsina 1 (partido de Patagones, provincia de Buenos Aires, Argentina): estudio de la estructura sexual y etaria de 4 entierros secundarios. Aportes para el conocimiento de las prácticas mortuorias en la cuenca inferior del Río Colorado". Facultad de Ciencias Sociales, Universidad Nacional del Centro de la Provincia de Buenos Aires, Argentina. Tesis de Licenciatura inédita. Biblioteca de la Faculta de Ciencias Sociales (UNCPBA).

Bayala, P. 2010. El registro bioarqueológico del sitio Paso Alsina 1 (curso inferior del río Colorado, provincia de Buenos Aires). Editado por M. Berón, L. Luna, M. Bonomo, C. Montalvo, C. Aranda y M. Carrera Aizpitarte, Mamul Mapu. Pasado y presente desde la arqueología pampeana, pp. 123-136. Libros del Espinillo, Buenos Aires.

Bayón, C., N. Flegenheimer y A. Pupio. 2006. Planes sociales en el abastecimiento y traslado de roca en la Pampa Bonaerense en el Holoceno temprano y tardío. Relaciones de la Sociedad Argentina de Antropología, XXXI: 19-45.

Bayón, C., G. Martínez, G. Armentano y C. Scabuzzo. 2004. Arqueología del Valle inferior del Río Colorado. Sitio La Primavera. Intersecciones en Antropología, 5: 39-55.

Bayón, C. A. Pupio, R. Frontini, R. Vecchi y C. Scabuzzo. 2010. Localidad arqueológica Paso Mayor: nuevos estudios 40 años después. Intersecciones en Antropología, 11: 115-128.

Bernal, V., P. González, S. Pérez y H. Pucciarelli. 2008. Entierros humanos del noreste de patagonia: nuevos fechados radiocarbónicos. Magallania, 36(2): 175-183.

Bridges, P. 1992. Prehistoric arthritis in the Americas. Annual Review of Anthropology, 21:67-91.

Buikstra, J. E., D. H. Ubelaker. 1994. Standards for Data Collection from Human Skeletal Remains. Arkansas Archaeological Survey Research Series No 44, Arkansas. Capasso, L., K. Kennedy y C. Wilczak. 1999. Atlas of occupational markers on human remains. Edigrafital S.P.A., Teramo.

Chapman, N. 1997. Evidence for Spanish influence in activity induced musculoskeletal stress markers at Pecos Pueblo. International Journal of Osteoarchaeology, 7: 497-506.

Flensborg, G. 2008. "Análisis paleopatológico en el sitio Paso Alsina 1. Primeros resultados sobre la salud de las sociedades cazadoras-recolectoras del valle inferior del río Colorado durante el Holoceno tardío final". Facultad de Ciencias Sociales. Olavarría, Universidad Nacional del Centro de la Provincia de Buenos Aires, Argentina. Tesis de Licenciatura inédita. En Biblioteca de la Faculta de Ciencias Sociales (UNCPBA).

Flensborg, G. 2010. Análisis paleopatológico en el sitio Paso Alsina 1. Primeros resultados sobre la salud de las sociedades cazadoras-recolectoras del valle inferior del río Colorado durante el Holoceno tardío final. Editado por M. Berón, L. Luna, M. Bonomo, C. Montalvo, C. Aranda y M. Carrera Aizpitarte, Mamul Mapu. Pasado y presente desde la arqueología pampeana, pp. 165-180. Libros del Espinillo, Buenos Aires.

Frontini, R. 2012. "El aprovechamiento de animales en valles fluviales y lagunas del sur bonaerense durante el Holoceno". Facultad de Filosofía y Letras, Universidad de Buenos Aires, Argentina, 367 pp. Tesis Doctoral inédita. Biblioteca del Instituto de Arqueología, UBA, Buenos Aires.

González, M. 2010. Huellas de corte y análisis contextual en restos óseos humanos de la cuenca inferior del río Colorado: implicaciones para el entendimiento de las prácticas mortuorias. Editado por M. Berón, L. Luna, M. Bonomo, C. Montalvo, C. Aranda y M. Carrera Aizpitarte, Mamul Mapu. Pasado y presente desde la arqueología pampeana, pp. 193-210. Libros del Espinillo, Buenos Aires.

Guichón, R., S. Muñoz y L. Borrero. 2000. Datos para una tafonomía de restos óseos humanos en Bahía San Sebastián, Tierra del Fuego. Relaciones de la Sociedad Argentina de Antropología, XXV: 297-311.

Hawkey, D. 1998. Disability, compassion and the skeletal record: using musculoskeletal stress markers to construct an osteobiography from early New Mexico. International Journal of Osteoarchaeology, 8: 326-340.

Hawkey, D., C. Merbs. 1995. Activity-induced musculoskeletal stress markers (MSM) and subsistence strategy among ancient Hudson Bay Eskimos. International Journal of Osteoarchaeology, 5: 324-338.

Jurmain, R. 1980. The Pattern of Involvement of Appendicular Degenerative Joint Disease. American Journal of Physical Anthropology, 53:143-150.

Kennedy, K. 1989. Skeletal markers of occupational stress. 
Editado por M. Iscan y K. Kennedy, Reconstruction of Life from the Skeleton, pp. 129-160. Alan R. Liss, Nueva York.

La Nueva Provincia. 1963. Arqueología en nuestra región. Resultados de una expedición del Museo Histórico bahiense. La Nueva Provincia, 6 de Mayo de 1963: 2.

Larsen, C. 1997. Bioarchaeology: Interpreting behavior from the human skeleton. Cambridge University Press, Cambridge.

Lessa, A. 2004. Arqueologia da agressividade humana: a violência sob uma perspectiva paleoepidemiológica. História, Ciências, Saúde, 11(2): 279-96.

L'Heureux, G. 2000. Estudios comparativos de indicadores de adecuación fisiológica y salud bucal en muestras de restos humanos del Sudeste de la Región Pampeana. Relaciones de la Sociedad Argentina de Antropología, XXV: 51-74.

Liberman, D., D. Raichlen, H. Pontzer, D. Bramble y E. Cutright-Smith. 2006. The human gluteus maximus and its role in running. Journal of Experimental Biology, 209: 2143-2155.

Luna, L. 2008. Estructura demográfica y relaciones biológicas de cazadores recolectores en un ambiente de desierto. Sitio Chenque I (Parque Nacional Lihué Calel, provincia de La Pampa, Argentina). BAR Internacional Series, Oxford.

Madrid, P., G. Politis. 1991. Estudios arqueológicos y paleoambientales en la Región Pampeana: un enfoque multidisciplinario del sitio La Toma. Actas XI Congreso Nacional de Arqueología Chilena, pp. 131-153. Santiago de Chile.

Martínez, G. 2010. Entierros humanos en lugares sagrados y domésticos durante el Holoceno tardío: el registro bioarqueológico del curso inferior del río Colorado (provincia de Buenos Aires, Argentina). Revista Werkén, (13): 145-160.

Martínez, G., M. Figuerero Torres. 2000. Sitio arqueológico La Petrona (Partido de Villarino, Provincia de Buenos Aires): análisis de la modalidad de entierro en el área sur pampeana. Relaciones de la Sociedad Argentina de Antropología, XXV: 227-248.

Martínez, G., G. Flensborg, P. Bayala y R. Lopéz. 2007. Análisis de la composición anatómica, sexo y edad de los entierros secundarios del sitio Paso Alsina 1 (Pdo. de Patagones, Pcia. de Buenos Aires). Editado por C. Bayón, N. Flegenheimer, M. I. González y M. Frere, Arqueología en las Pampas, pp. 41-58. Sociedad Argentina de Antropología, Buenos Aires.
Martínez, G., A. Zangrando y L. Prates. 2009. Isotopic ecology and human paleodiets in the lower basin of the Colorado River, Buenos Aires province, Argentina. International Journal of Osteoarchaeology, 19:281-296.

Méndez, G y S. Salceda. 1989. Provincia de Buenos Aires: Ensayo de clasificación de sus antiguos pobladores. Revista del Museo de La Plata (N.S.) Antropología, IX (68): 93-105.

Mendonça de Souza, S. 1992. Traumatismos vertebrais como indicadores de atividade fisica na populaçao da Furna do Estrago, Pernambuco, Brasil. Editado por A. Araújo y L. Ferreira, Paleopatologia e PaleoepidemiologiaEstudos Multidisciplinares, pp. 123-140. Panorama ENSP, Río de Janeiro.

Merbs, C. 1983. Patterns of Activity-Induced Pathology in a Canadian Inuit Population. Archaeological survey of Canada, Ottawa.

Molleson, T. 1994. The eloquent bones of Abu Hureya. Scientific American, 271(2): 70-75.

Neves, W. 1984. Estilo de vida e osteobigrafia: a reconstituição do comportamento pelos osso humanos. Revista Pré-História, 6: 287-291.

Ortner, D., W. Putschar. 1981. Identification of pathological conditions in human skeleton remains. Smithsonian Contributions to Anthropology 28. Smithsonian Institution, Washington.

Perez, I. 2002. Variabilidad temporal en la morfología craneofacial en muestras de restos humanos del sudeste de la región pampeana: implicaciones para la discusión del poblamiento regional durante el Holoceno. Editado por D. Mazzanti, M. Berón y F. Oliva, Del Mar a los Salitrales. Diez Mil Años de Historia Pampeana en el Umbral del Tercer Milenio, pp.155-170. UNMdP, Mar del Plata.

Politis, G. 1984. "Arqueología del Área Interserrana Bonaerense". Facultad de Ciencias Naturales y Museo, Universidad Nacional de La Plata, Argentina, 310 pp. Tesis Doctoral inédita. Biblioteca de la Facultad de Ciencias Naturales y Museo.

Politis, G., P. Madrid. 2001. Arqueología Pampeana: Estado actual y perspectivas. Editado por E. Berberián y A. Nielsen, Historia Argentina Prehispánica, pp.737-813. Brujas, Argentina.

Politis, G., C. Scabuzzo y R. Tykot. 2009. An approach to prehispanic diets in the pampas during early/middle Holocene. International Journal of Osteoarchaeology, 19: 266-280.

Rodrigues Carvalho, C. 2004. "Marcadores de Estresse 
Ocupacional em Populações Sambaquieiras do Litoral Fluminense". Fundación Osvaldo Cruz, Brasil, 212 pp. Tesis Doctoral inédita. Biblioteca Central del Museo Nacional de Rio de Janeiro.

Salceda, S., M. Méndez. 1990. Los restos óseos de Paso Vanoli (Pcia. de Buenos Aires). Archeion. Archivo del Laboratorio de Antropología Biológica, 1: 3-24.

Santiago, F., M. Salemme, J. Suby y R. Guichón. 2012. Restos humanos en el norte de Tierra del Fuego. Aspectos contextuales, dietarios y paleopatológicos. Intersecciones en Antropología 12: 147-162.

Scabuzzo, C. 2010. "Actividades, patologías y nutrición de los cazadores recolectores pampeanos". Facultad de Ciencias Naturales y Museo, Universidad Nacional de La Plata, Argentina, 292 pp. Tesis Doctoral inédita. Biblioteca de la Facultad de Ciencias Naturales y Museo.

Scabuzzo, C. 2012. Estudios Bioarqueológicos de Marcadores de Estrés Ocupacional en Cazadores Recolectores Pampeanos del Holoceno temprano medio. Análisis de la Serie Esqueletal de Arroyo Seco 2. Revista Argentina de Antropología Biológica, 14(1): 17-31.

Scabuzzo, C., G. Politis. 2011. Los entierros secundarios del Holoceno temprano y medio en la región pampeana.
Nuevos datos del sitio Arroyo Seco 2. Cazadores Recolectores del Cono Sur, 4: 135-155.

Steen, S., R. Lane. 1998. Evaluation of habitual activities among two Alaskan Eskimo populations based on musculoskeletal stress markers. International Journal of Osteoarchaeology, 8:341-353.

Teixeira, A. S. M. 2004. "Estudo dos marcadores de estresse musculoesquelético em restos esqueleticos dos habitantes do sambaqui de Cabeçuda, Laguna, SC. Uma abordagem biocultural." Instituto de Ciências Biomédicas de la Universidade Federal do Rio de Janeiro, Brasil. Tesis de Maestria inédita. Biblioteca Central del Museo Nacional de Rio de Janeiro.

Ubelaker, D. 1979. Skeletal evidence for kneeling in Prehistoric Ecuador. American Journal of Physical Anthropology, 51:679-686.

Vecchi, R. 2011. "Bolas de boleadora en los grupos cazadores-recolectores de la Pampa bonaerense". Facultad de Filosofía y Letras, Universidad de Buenos Aires, Argentina, 360 pp. Tesis Doctoral inédita. Biblioteca del Instituto de Arqueología, UBA, Buenos Aires.

White, T., P. Folkens. 2005. The Human Bones Manual. Elsevier Academic Press, Londres. 